\title{
Young and Fresnel without sodium lamp
}

\section{Young y Fresnel sin lámpara de sodio}

\author{
Sebastián Jarabo ${ }^{S^{*}}$ \\ Departamento de Física Aplicada, Facultad de Ciencias, Universidad de Zaragoza \\ (*) E-mail: sjarabo@unizar.es S: miembro de SEDOPTICA / SEDOPTICA member \\ Received / Recibido: 28/07/2015 Accepted / Aceptado: 07/09/2015 \\ DOI: $10.7149 /$ OPA.48.3.243
}

\begin{abstract}
:
The well-known experiment of Young about interferences by means of a Fresnel biprism to obtain the double-slit is usually made using quasi-monochromatic sources: a sodium spectral lamp, a laser, etc. Besides, a monochromatic source is also assumed in order to develop the theoretical explanation of the experiment. Then, some experimental details are lost, as the dependence of the fringe-to-fringe spacing on the wavelength. Moreover, a much more dangerous negative effect is originated, since some students come to the following false conclusion: interference phenomena only are originated by monochromatic sources. In this paper, an easy modification of this experiment is shown. Thus, by employing a white source and RGB filters, it is possible to observe interference fringes with polychromatic light and verify that its period raises with the wavelength.
\end{abstract}

Key words: Interferences, double-slit, RGB filters

\section{RESUMEN:}

El clásico experimento de las franjas interferenciales de Young empleando un biprisma de Fresnel para obtener la doble rendija suele realizarse con fuentes casi monocromáticas: una lámpara espectral de sodio, un láser, etc. Incluso la explicación teórica suele asumir que la fuente es monocromática. Esto impide observar algunos aspectos del experimento, como que la interfranja aumenta con la longitud de onda. Además, origina un efecto negativo mucho más grave, ya que induce a algunos estudiantes a concluir de forma errónea que las interferencias sólo se producen cuando se emplean fuentes monocromáticas. En este artículo se muestra una sencilla modificación del experimento empleando luz blanca y filtros RGB, la cual permite observar claramente interferencias con luz policromática y verificar que la interfranja aumenta con la longitud de onda.

Palabras clave: Interferencias, doble rendija, filtros RGB

\section{REFERENCES AND LINKS / REFERENCIAS Y ENLACES}

[1] M. Born, E. Wolf, Principles of optics: Electromagnetic theory of propagation, interference and diffraction of light. Cambridge, Cambridge University Press (1999).

[2] R. W. Ditchburn, Light, New York, Dover Publications Inc. (1991).

[3] J. Casas, Óptica. Zaragoza, Librería General (1994).

[4] F. Carreño Sánchez, M. Antón Revilla, J. M. Ezquerro Rodríguez, O. Gómez Calderón, Experiencias de Óptica Física. Madrid, Editorial Complutense (2001).

[5] R. P. Crease, El prisma y el péndulo. Los diez experimentos más bellos de la ciencia. Barcelona, Crítica (2006).

[6] E. Hecht, Óptica. Madrid, Addison Wesley Iberoamericana (2000).

[7] M. L. Calvo Padilla, "Coherencia óptica," Temas Investigación y Ciencia 6, 46-53 (1996). 


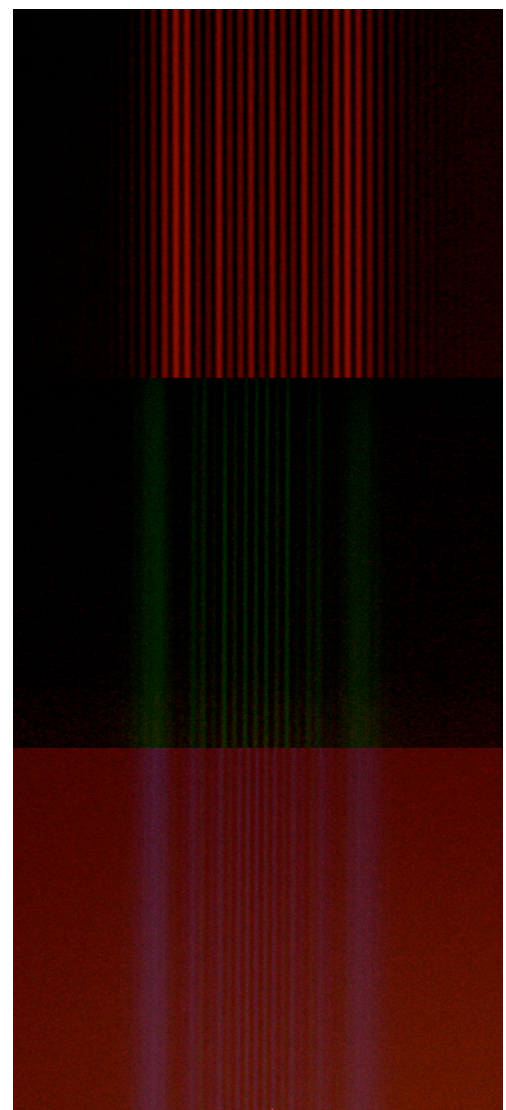

Fig.1. Franjas de Young con fuente policromática filtrada mediante filtros RGB. La anchura de la interfranja aumenta con la longitud de onda.

\section{Introducción}

La observación de las franjas de interferencia de Young creando la doble rendija mediante el biprisma de Fresnel es un clásico, un experimento que no puede faltar en ningún curso de Óptica Física [1-4]. Experimento sencillo, pero fundamental, ya que en su momento sirvió para demostrar el comportamiento ondulatorio de la luz. No en vano, fue recogido en su libro por Robert P. Crease como uno de los experimentos más bellos de la Ciencia [5]. Básicamente, el frente de onda procedente de una rendija iluminada de forma uniforme es dividido mediante un biprisma de Fresnel cuya arista es paralela a la rendija. A partir del biprisma, es como si la luz procediese de dos rendijas paralelas entre sí. Es decir, el biprisma crea dos imágenes virtuales de la rendija (rendijas virtuales). Los haces de luz procedentes de ambas fuentes virtuales interferirán entre sí de forma que, en un plano perpendicular a la dirección de propagación que esté suficientemente alejado del biprisma, la distribución de intensidad se mantiene constante en la dirección paralela a la rendija, pero varía de forma periódica en la dirección perpendicular a ésta (coordenada $x$; la posición de la arista nos fija el origen de coordenadas).

Si consideramos que el haz de luz es monocromático con una longitud de onda en el vacío $\lambda_{0}$, la intensidad varía conforme nos desplazamos sobre el plano de observación siguiendo una función coseno cuadrado

$$
I(x) \propto \cos ^{2}\left(\frac{\pi}{\Delta x} x\right), \Delta x=\frac{D}{d} \lambda_{0},
$$

siendo $d$ la distancia entre ambas rendijas virtuales y $D$ la distancia desde la rendija hasta el plano de observación. Es decir, se produce una distribución periódica de máximos y mínimos de intensidad con contraste unidad y un periodo $\Delta x$. Debido al comportamiento de nuestro ojo como detector, cuando se observa la distribución de intensidad sobre dicho plano, vemos una distribución luminosa en forma de franjas paralelas a la dirección de la rendija. La separación entre dos franjas consecutivas (interfranja) viene dada por el periodo de la función coseno cuadrado. 
Para obtener un buen contraste en las franjas de interferencia es fundamental que la rendija y la arista del biprisma estén bien paralelas entre así. Además, si la rendija se ilumina con una lámpara, es imprescindible que la rendija esté suficientemente cerrada debido a la baja coherencia espacial de la fuente $[3,7]$. Entonces, en la figura interferencial se superpone la variación de intensidad debida a la difracción que produce la rendija. Como también se reduce mucho la intensidad que llega al plano de observación, es necesario emplear una lámpara con gran potencia de emisión. Usualmente, se emplea una lámpara espectral de sodio [7]. Otra posibilidad es iluminar la rendija con un haz láser (expandido) [4], lo que evita los problemas anteriores y produce una distribución de franjas muy espectacular. Ejemplos de franjas de interferencia obtenidos con una lámpara de sodio y con un láser de estado sólido son mostrados en las figuras 2 y 3 , respectivamente.

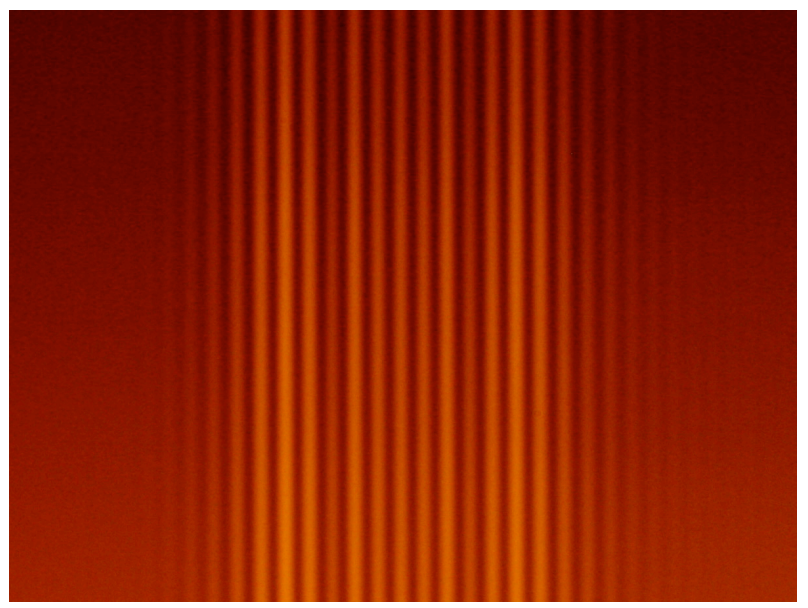

Fig.2. Franjas de Young con una lámpara espectral de sodio (589 nm).

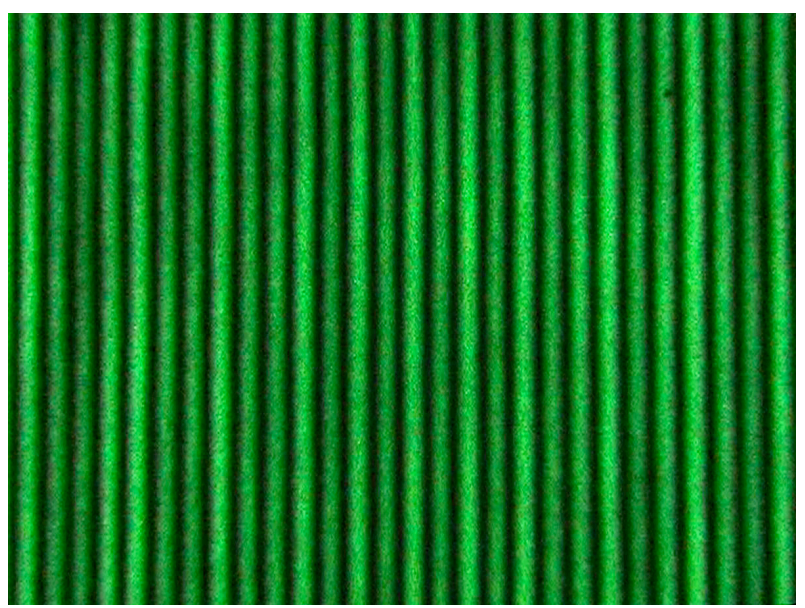

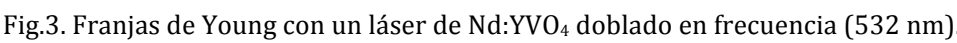

Como las dos fuentes que se emplean habitualmente son prácticamente monocromáticas, parte de la fenomenología del experimento se pierde: ni puede apreciarse la dependencia de la interfranja con la longitud de onda ni puede observarse que en $\mathrm{x}=0$ hay máximo interferencial independientemente de la longitud de onda. Pero además, como casi siempre se deducen las ecuaciones considerando luz monocromática se produce una percepción errónea por parte del estudiante, que llega a la conclusión de que si la fuente no es casi monocromática, no se producen interferencias. En realidad, el fenómeno interferencial siempre está ahí, aunque se manifieste con mejor o peor contraste. Incluso con luz blanca, tal como describen la mayoría de los textos de Óptica $[1,3,6]$, aunque no suelen mostrar imágenes. Es fácil comprobarlo experimentalmente empleando como fuente policrómatica un foco halógeno, tal como se muestra en la figura 4, donde las franjas de interferencia aparecen en diferentes colores ya que la interfranja varía con la longitud de onda. La franja central no se ve blanca ya que la intensidad de luz es 
muy baja y la CCD de la cámara fotográfica modifica el color. Sin embargo, mirando directamente las franjas, la franja central aparece de color blanquecino.

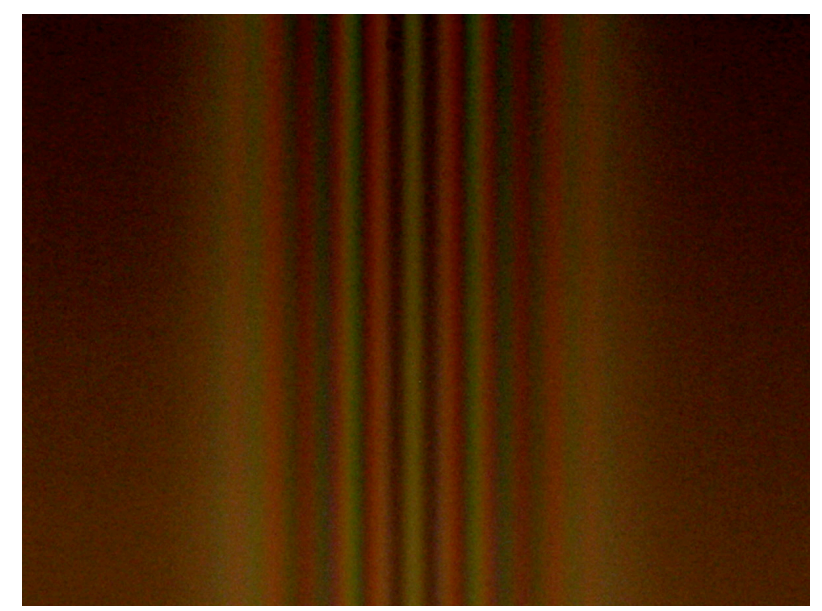

Fig.4. Franjas de Young con una fuente policromática (foco halógeno).

No obstante, las observaciones con luz blanca no son lo suficientemente claras y resulta necesario llevar a cabo demostraciones en las que se aprecie claramente esta dependencia. La solución más simple consiste en emplear un montaje típico de franjas de Young con biprisma de Fresnel iluminando con una fuente blanca. Entonces, mientras se observan las franjas (inicialmente, aparecen coloreadas) se interponen sucesivamente filtros rojo, verde y azul, bien entre la rendija y el biprisma, o bien justo antes del ocular de observación. Con independencia de la posición del filtro conseguimos el mismo efecto: limitar la anchura espectral de la fuente y centrar su espectro en tres longitudes de onda diferentes. El estudiante podrá observar claramente las franjas empleando luz roja, verde o azul, apreciando cómo la interfranja varía con la longitud de onda. Además, como estamos empleando luz poco monocromática, se destierra el error de considerar que se necesita luz monocromática para que se produzcan fenómenos interferenciales. El efecto observado se pretende ilustrar con la figura 1, en la que se muestran las imágenes de interferencia que se observan con los tres filtros citados.

\section{Así se hizo}

Se dispuso un montaje experimental típico de doble rendija mediante biprisma de Fresnel (figura 5). Una rendija de anchura variable fue iluminada con un foco halógeno convencional. Mediante un biprisma de Fresnel colocado frente a la rendija se divide el frente de onda y se observan las interferencias en un plano suficientemente alejado de la rendija a través de una cámara réflex sin objetivo. Justo delante de la cámara se interpone un filtro de color rojo. Se ajusta la anchura de la rendija y se orienta para que quede bien paralela a la arista del biprisma, de forma que se observen bien nítidas las franjas de interferencia a través del visor de la cámara réflex. Al disparar la cámara, su espejo se recoge y las franjas de interferencia se formarán sobre la CCD de la cámara. Se repite el proceso empleando un filtro verde y un filtro azul. Los filtros empleados corresponden a un conjunto de tres filtros RGB, comprados a la empresa Thorlabs. Su factor de transmisión se representa en la figura 6. Como separan las zonas roja, verde y azul del espectro podemos observar la anchura de la interfranja para tres zonas espectrales diferentes, es decir, para tres longitudes de onda diferentes. No obstante, hay que tener presente que el espectro final que estamos utilizando sería el producto del espectro de potencia del foco, de la transmisión espectral del filtro y de la respuesta espectral de la CCD. Las fotografías de las figuras 2, 3 y 4 fueron obtenidas por el autor empleando esta misma técnica. 


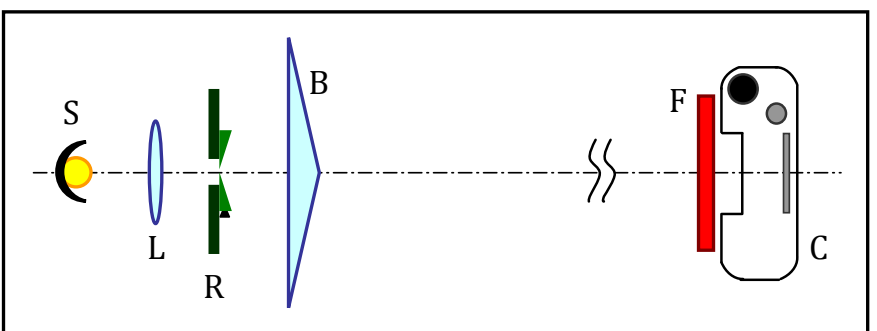

Fig.5. Esquema del montaje experimental: foco halógeno (S), lente convergente auxiliar (L) para concentrar luz sobre la rendija en caso que sea necesario, rendija de anchura variable $(R)$, biprisma de Fresnel (B), filtro espectral (F) rojo, verde o azul, cámara digital réflex sin objetivo (C).

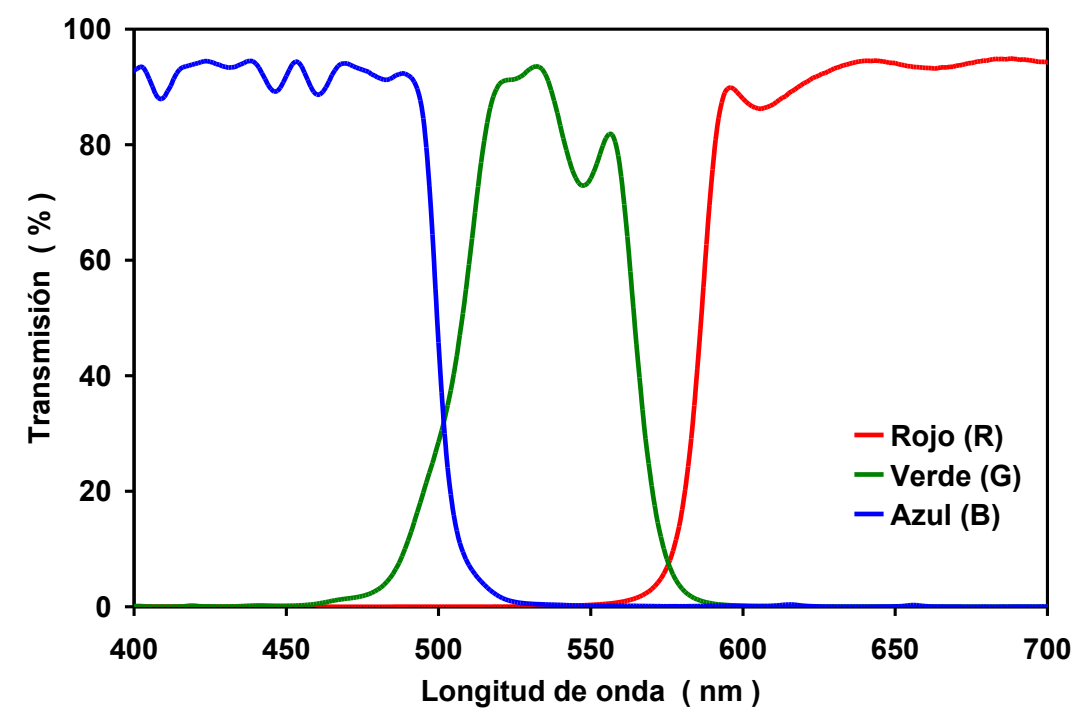

Fig.6. Factor de transmisión espectral de los filtros espectrales empleados (RGB).

\section{Resultado: la fotografía}

La fotografía de la figura 1 ilustra lo que se observa cuando se forman franjas de Young con una fuente policromática filtrada mediante filtros RGB. De esta forma, se observa cómo la interfranja aumenta con la longitud de onda, tal como indica la ecuación (1). A pesar de que las tres fuentes empleadas tienen un espectro muy ancho (decenas de $\mathrm{nm}$ ), las franjas de interferencia pueden apreciarse con un buen contraste para los filtros rojo y verde y con un contraste aceptable para el filtro azul. Es necesario tener en cuenta que los focos halógenos emiten poca potencia en la zona azul del espectro y, además, la CCD suele tener una respuesta espectral bastante pobre en dicha zona.

Es una demostración clara de que no es necesario utilizar fuentes monocromáticas, como la lámpara espectral de sodio, para realizar un experimento sobre interferencias, evitando así la percepción errónea que adquieren algunos estudiantes tras realizar este tipo de experimentos. 